\title{
Podridão apical e escaldadura em frutos de pimentão submetidos a estresse hídrico e doses de silício
}

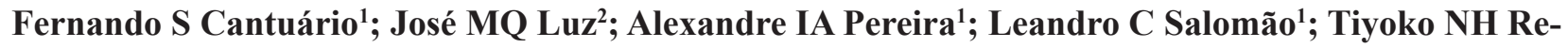 \\ bouças $^{3}$ \\ ${ }^{1}$ IF Goiano, Rod. Geraldo Silva Nascimento km 2,5; 75790-000 Urutaí-GO; fernandoscantuario@hotmail.com; aiapereira@yahoo.com. \\ br; salomaoleandro@gmail.com; ㄹFU-ICIAG, Bloco 2E, Campus Umuarama, 38400-902 Uberlândia-MG; jmagno@umuarama.ufu.br; \\ ${ }^{3}$ UESB, Estrada do Bem Querer km 04, 45083-900 Vitoria da Conquista-BA; tiyoko@uesb.edu.br
}

\begin{abstract}
RESUMO
As respostas da planta de pimentão submetida ao estresse hídrico são pouco conhecidas. A busca por indutores de resistência ao estresse hídrico (como a adubação silicatada) pode ser uma importante medida para reduzir os efeitos negativos desse estresse abiótico. Dessa forma, o objetivo desse estudo foi testar a hipótese de que a adubação silicatada aplicada via foliar pode atenuar a incidência de anomalias causadas pelo estresse hídrico na qualidade dos frutos de pimentão. $\mathrm{O}$ experimento foi conduzido em ambiente protegido na área experimental de olericultura do Instituto Federal Goiano, câmpus Urutaí-GO. O delineamento utilizado foi em blocos casualizados, com parcela subdividida no espaço, em esquema fatorial $3 \times 4$, sendo três doses de silício $\left(0,0,4,3,8,6 \mathrm{~kg} \mathrm{ha}^{-1}\right)$ na forma de silicato de potássio $\left(\mathrm{K}_{2} \mathrm{SiO}_{3}\right)$ e quatro tensões de água no solo de aproximadamente $-15,-25,-35$ e $-45 \mathrm{kPa}$, totalizando 12 tratamentos, com quatro repetições. Os níveis de água no solo para a produção do pimentão, cultivar híbrido Magali-R, variaram desde a suficiente oferta de água até o déficit hídrico e, também, valores intermediários. Vinte e uma aplicações foliares de silicato de potássio foram efetuadas semanalmente ao longo do experimento. $\mathrm{O}$ aumento da tensão de água no solo e a ausência do silicato de potássio estiveram correlacionados com a incidência da podridão apical nos frutos. Nenhum dos dois fatores estudados influenciou a incidência de escaldadura nos frutos de pimentão. No total foram 129 frutos de pimentão com podridão apical e 70 com escaldadura, representando $2,14 \%$ e $1,16 \%$ do total de frutos colhidos.
\end{abstract}

Palavras-chave: Capsicum annuum, deficiência hídrica, adubação silicatada, anomalias fisiológicas.

\section{ABSTRACT \\ Blossom-end rot and scald in fruits of sweet pepper submitted to water stress and silicon rates}

The response of sweet pepper plants submitted to water stress is little known. The search for inducing resistance to water stress (such as silicon fertilization) can be an important step to reduce the negative effects of this abiotic stress. Thus, the aim of this study was to test the hypothesis that silicon fertilization through foliar applications can mitigate the incidence of anomalies caused by water stress on fruit quality of sweet pepper. The experiment was carried out in a greenhouse at the experimental horticulture area of the Instituto Federal Goiano, câmpus Urutaí, Goiás state, Brazil. The experimental design was randomized complete blocks, in a $3 \times 4$ factorial design, with split plots in space, and three levels of silicon $\left(0.0,4.3,8.6 \mathrm{~kg} \mathrm{ha}^{-1}\right)$ in the form of potassium silicate $\left(\mathrm{K}_{2} \mathrm{SiO}_{3}\right)$ and four soil water tensions of approximately $-15,-25,-35$ and $-45 \mathrm{kPa}$, totaling 12 treatments with four replications. Water levels in the soil for the production of sweet pepper cultivar Magali-R, ranged from sufficient supply of water to the drought and also intermediate values. Twenty one foliar applications of potassium silicate were used weekly throughout the experiment. Increased soil water tension and the absence of potassium silicate were correlated with the incidence of blossom-end in fruits. The incidence of scald in sweet pepper fruits was not influenced by any of the two studied factors. A total of 129 fruits of sweet pepper presented blossom-end rot and 70 fruit presented scald, representing $2.14 \%$ and $1.16 \%$ of total harvested fruit.

Keywords: Capsicum annuum, water deficit, silicate fertilization, physiological anomalies.

\section{(Recebido para publicação em 21 de dezembro de 2012; aceito em 27 de março de 2014)} (Received on December 21, 2012; accepted on March 27, 2014)

$\mathrm{O}$ pimentão (Capsicum annuum) é uma Solanácea, tipicamente de origem americana, ocorrendo formas silvestres no México, América Central e América do Sul (Souza et al., 2011). O Brasil está entre os principais países produtores de pimentão (Filgueira, 2008). As maiores áreas de produção estão localizadas no Sudeste, sendo esta a principal região produtora do país (Souza et al., 2011).

A deficiência de água na cultura do pimentão é um dos fatores limitantes para a obtenção de elevada produtividade e qualidade dos frutos, tanto em ambiente protegido ou em campo. A falta de água em plantas de pimentão durante a fase inicial de frutificação pode restringir a translocação de nutrientes como o cálcio e reduzir a cobertura foliar, contribuindo, respectivamente, para o surgimento das anomalias fisiológicas podridão apical e escaldadura, causando perdas significativas na qualidade e produção dos frutos (Hartz et al., 2008).

A podridão apical caracteriza-se por uma mancha negra, dura e seca na extremidade apical, bem visível desde o inicio da formação dos frutos, causada pela deficiência de cálcio, que pode estar associada à deficiência hídrica e ao excesso de sais no solo. A escaldadura ocorre quando o fruto é exposto à luz solar intensa, próximo ao período de colheita. A região afetada torna-se esbranquiçada e enrugada. As principais causas estão associadas à redução da cobertura foliar e à consequente exposição dos frutos a forte radiação solar, 
o que pode ser originado pelo ataque de pragas e doenças e pela falta de água na fase de produção. Portanto, são comuns os sintomas dessas anomalias em condições de desequilíbrio nutricional e deficiência hídrica na solução do solo e/ou na planta (Saure, 2001; Filgueira, 2008; Souza et al., 2011 ).

O uso de silício ( $\mathrm{Si}$ ) como um elemento capaz de reduzir os efeitos negativos dos estresses abióticos em plantas vem despertando o interesse na área da produção vegetal. Os efeitos benéficos do Si podem ser divididos em dois grupos: físicos e fisiológicos. Os benefícios físicos estão relacionados ao acúmulo do Si na parede celular das plantas, formando uma barreira física à redução da perda de água, melhorando a arquitetura das plantas (Korndörfer et al., 2002). Segundo Ma \& Yamaji (2006), a maioria dos efeitos benéficos do $\mathrm{Si}$ em reduzir o estresse hídrico é atribuída à deposição de Si na parede celular de raízes, folhas e caules. Os benefícios fisiológicos estão relacionados com aumento da atividade fotossintética, resistência ao ataque de fitopatógenos e pragas e da tolerância das plantas à seca, devido ao $\mathrm{Si}$ induzir uma série de reações metabólicas nas plantas resultando na formação de compostos como fitoalexinas, ligininas (Menzies et al., 1991; Vidhyasekaran, 1997; Pozza et al., 2004), potencializar atividades de enzimas como peroxidases, polifenoloxidases e quintinases (Liang \& Sun, 2005), aumentar a ação de defesas antioxidativas, reduzir o dano oxidativo de moléculas funcionais em membranas e aumentar a resistência estomática, no teor de peróxido de hidrogênio e prolina (Gong et al., 2005; Gunes et al., 2007; Crusciol et al., 2009).

Diversos trabalhos demonstram a eficiência na aplicação de Si via solo e na solução hidropônica para plantas mono e dicotiledôneas no que se refere à resistência contra patógenos, anomalias fisiológicas, seca e também no incremento da produtividade (Liang et al., 2005; Luz et al., 2006; Nolla et al., 2012). A aplicação foliar de Si é considerada menos eficiente que aplicação via solo ou solução hidropônica, porém, vários autores comprovam suas vantagens em diversas culturas (Luz et al., 2006; Rodrigues et al., 2007; Buck et al., 2008; Rodrigues et al., 2010), provavelmente pelo fato desse mineral acumular-se na superfície foliar, servindo como barreira física e tendo um importante papel na regulação osmótica, diminuindo o estresse causado pela falta de água nas plantas. $\mathrm{O}$ interesse em utilizar o Si via adubação foliar no presente trabalho foi o de avaliar as vantagens que essa técnica tem proporcionado seja pela sua praticidade, possibilidade de utilização de doses menores e também pela sua adaptabilidade ao equipamento de aplicação normalmente utilizado pelos produtores, considerando inclusive os plantios em sequeiro.

O objetivo desse estudo foi avaliar a hipótese de que a adubação silicatada aplicada via foliar pode atenuar os efeitos negativos causados pelo estresse hídrico na qualidade dos frutos de pimentão.

\section{MATERIAL E MÉTODOS}

O experimento foi instalado no Instituto Federal Goiano (IF Goiano), câmpus Urutaí-GO (17²9'10"S, 48 12 '38'O, 697m de altitude). O clima da região é classificado como tropical de altitude, com inverno seco e verão chuvoso, do tipo Cwb pela classificação de Köppen. O solo da área experimental foi classificado como Latossolo Vermelho Amarelo distrófico, com textura franco arenosa.

O experimento foi instalado durante os meses de junho de 2011 a janeiro de 2012, conduzido em ambiente protegido do tipo arco simples, com orientação leste-oeste e estrutura metálica, dimensões de $30 \mathrm{~m}$ de comprimento, $7 \mathrm{~m}$ de largura, pé-direito de 3,0 $\mathrm{m}$ e altura de arco de 1,2 m, coberta com filme de polietileno de baixa densidade (PEBD) de 0,15 mm de espessura, laterais constituídas de telado e com cortinas móveis (fechadas apenas nos dias chuvosos). No interior do ambiente foi instalado um sistema de nebulização, com nebulizadores destinados à climatização do ambiente, possuindo micro gotas de 40 micra, com sistema anti-gotas.

O delineamento experimental utilizado foi em blocos casualizados com parcelas subdivididas no espaço, em esquema fatorial $3 \times 4$, sendo o primeiro fator a concentração de silício, com três níveis $\left(0,0,4,3\right.$ e $\left.8,6 \mathrm{~kg} \mathrm{ha}^{-1}\right)$ e o segundo, a tensão de água no solo, com quatro níveis (aproximadamente -15 , $-25,-35$ e $-45 \mathrm{kPa}$ ), totalizando doze tratamentos, com quatro repetições. As tensões de água no solo foram alocadas nas parcelas e as concentrações de silício na forma silicato de potássio nas subparcelas. O experimento foi constituído por quatro canteiros úteis (blocos) com duas linhas de plantio por canteiro. Nas laterais do ambiente protegido (bordadura) foram instalados dois canteiros com uma linha de plantio.

A adubação seguiu recomendações de Trani \& Carrijo (2004) e Trani (2007) levando em consideração a análise de solo realizada no laboratório da UFU em Uberlândia, feita nos sulcos de plantio 15 dias antes do transplantio das mudas ao local definitivo e contou com a aplicação de $40 \mathrm{~kg} \mathrm{ha}^{-1}$ de N, $60 \mathrm{~kg} \mathrm{ha}^{-1}$ de $\mathrm{P}_{2} \mathrm{O}_{5}, 100 \mathrm{~kg} \mathrm{ha}^{-1}$ de $\mathrm{K}_{2} \mathrm{O}$ e $2 \mathrm{~kg} \mathrm{ha}^{-1}$ de $\mathrm{B}$ na forma de uréia, superfosfato simples, cloreto de potássio e bórax, respectivamente. A quantidade de $\mathrm{N}$ e $\mathrm{K}_{2} \mathrm{O}$ em cobertura foi de $120 \mathrm{~kg} \mathrm{ha}^{-1}$ para ambos os nutrientes; utilizou-se as fontes uréia ( $46 \%$ de $\mathrm{N})$ e cloreto de potássio branco $\left(60 \% \mathrm{~K}_{2} \mathrm{O}\right)$, parceladas e aplicadas em cinco fertirrigações ao longo do experimento.

A cultivar utilizada foi o híbrido Magali-R. A semeadura foi realizada em 20/06/2011, em bandejas de poliestireno expandido (isopor) com 128 células. Utilizou-se substrato comercial esterilizado. As mudas foram produzidas em casa de vegetação e foram transplantadas em 10/08/2011 em espaçamento de $0,8 \mathrm{~m}$ entre linhas e de $0,4 \mathrm{~m}$ entre plantas.

Utilizou-se um sistema de irrigação localizada por gotejamento. Cada linha de plantio recebeu uma linha lateral de irrigação de $16 \mathrm{~mm}$, com emissores espaçados entre si a $40 \mathrm{~cm}$. Os emissores apresentavam uma vazão de $1,4 \mathrm{~L} \mathrm{~h}^{-1}$, a pressão de serviço de 10 mca. O sistema de bombeamento era composto por conjunto motobomba de $1 \mathrm{cv}$. Logo após o sistema de bombeamento, foi instalado um injetor de fertilizante tipo Venturi, um filtro de tela de 120 mesh, registros 
e manômetros.

O manejo de irrigação foi realizado com o auxílio das curvas de retenção de água no solo. Dois tensiômetros de punção foram instalados em cada parcela experimental, totalizando trinta e dois tensiômetros. Para determinação da lâmina de irrigação nos primeiros 50 dias após o transplantio (DAT), foram escolhidos os tensiômetros instalados a $10 \mathrm{~cm}$ de distância da planta e $10 \mathrm{~cm}$ de profundidade, e para o restante do período experimental utilizaram-se os tensiômetros instalados na profundidade de $20 \mathrm{~cm}$. Portanto, nos primeiros 50 dias, os tensiômetros ficaram instalados nas profundidades de 10 e $20 \mathrm{~cm}$; após este período os tensiômetros instalados a $10 \mathrm{~cm}$ foram aprofundados para $40 \mathrm{~cm}$. A tomada de tensão dos tensiômetros foi realizada através de um tensímetro digital.

Aos primeiros 20 dias após o transplantio não houve diferenciação do manejo de irrigação para os tratamentos, com intuito de garantir o estabelecimento das plantas de pimentão, ocorrendo irrigações iguais em todas as parcelas. Após este período, deu-se inicio à diferenciação entre os tratamentos, nos quais as irrigações eram efetuadas quando as tensões atingissem, aproximadamente, $-15,-25,-35$ e $-45 \mathrm{kPa}$.

Para os tratamento de aplicação foliar, foi utilizado o produto silicato de potássio $\left(\mathrm{K}_{2} \mathrm{SiO}_{3}\right)$ líquido, solúvel em água, contendo $12,2 \%$ de silício e $15 \%$ de óxido de potássio, pH igual a 12 e densidade de $1,4 \mathrm{~g} \mathrm{dm}^{-3}$. As aplicações foram feitas nas subparcelas de acordo com os tratamentos $\left(0,0,4,3,8,6 \mathrm{~kg} \mathrm{ha}^{-1}\right.$ de $\mathrm{Si}$ ), com o auxilio de um pulverizador costal de ação manual de 20 L. Utilizou-se uma proteção de deriva para garantir a máxima precisão de aplicação. Foram realizadas aplicações semanais totalizando 21 aplicações, sendo o volume de calda utilizado de $600 \mathrm{~L} \mathrm{ha}^{-1}$. As doses de silicato de potássio em cada aplicação foram $0,0,1,68$ e $3,36 \mathrm{~kg} \mathrm{ha}^{-1} \mathrm{e}$ as concentrações de silício foram 0,0, 0,2049, $0,4098 \mathrm{~kg} \mathrm{ha}^{-1}$, totalizando $0,0,4,3,8,6$ $\mathrm{kg} \mathrm{ha}{ }^{-1}$ de $\mathrm{Si}$ ao final do experimento, respectivamente. As doses de Si usadas no presente trabalho assemelham-se às utilizadas por Gonçalves (2009) que trabalhou com adubação silicatada via foliar na cultura da batata.

Ao longo do ciclo da cultura do pimentão, foram realizados os tratos culturais necessários ao seu pleno desenvolvimento, como tutoramento, desbrota, capinas manuais e controle fitossanitário.

Foram realizadas cinco colheitas durante todo o período experimental, iniciando em 01/11/2011, aos 84 DAT, com intervalos médios de 17 dias. Os frutos foram colhidos em oito plantas úteis em cada subparcela (de um total de doze plantas por subparcela). Analisaram-se todos os frutos colhidos nas cinco colheitas, totalizando 6010 frutos, contabilizando os frutos com as anomalias fisiológicas podridão apical e escaldadura. A análise estatística foi feita com o uso da análise de variância (ANOVA). A normalidade foi verificada pelo teste de aderência de Lilliefors e, de forma complementar, visualmente pela simetria do histograma obtido pelo programa SAEG (Sistema de Análises Estatística e Genética; Ribeiro Junior \& Melo, 2009). De acordo com esse procedimento, as variáveis podridão apical e escaldadura não seguiram distribuição normal, sendo seus valores transformados em $\log (\mathrm{x}+1)$ pelo fato de que, nesses casos, os desvios padrões das amostras foram proporcionais às médias. Adicionalmente, utilizou-se o Coeficiente de Variação (CV) como indicativo para diagnosticar o acerto na escolha da transformação seguindo metodologia de Ferreira (2000). Para os dados transformados, a análise de variância e a comparação entre médias dos tratamentos foram realizadas na escala transformada. Porém, os resultados descritos para essas variáveis permaneceram em escala original. Após a verificação da significância (ou não) dos fatores em interação ou considerados isolados através da ANOVA com esquema fatorial, as médias foram comparadas utilizando o teste Tukey ao nível de $5 \%$ de probabilidade.

\section{RESULTADOS E DISCUSSÃO}

Um total de 129 e 70 frutos de pimentão foram acometidos com podridão apical e escaldadura, respectivamente, representando 2,14 e $1,16 \%$ do total de frutos colhidos ao longo de todo o período experimental. A interação entre a tensão de água no solo e o silicato de potássio não foi significativa para ambas anomalias fisiológicas e a incidência de frutos de pimentão com escaldadura não sofreu influência de nenhum dos dois fatores quando tratados de forma isolada. Todavia, a tensão de água no solo $(\mathrm{F}=4,49 ; \mathrm{p}=0,009)$ e a concentração de silício $(\mathrm{F}=5,81 ; \mathrm{p}=0,007)$ influenciaram significativamente a incidência de podridão apical nos frutos de pimentão.

Para esses casos, a menor quantidade de água no solo (maiores valores de tensão de água) e a ausência de aplicação

Tabela 1. Coeficiente de correlação entre a incidência das anomalias fisiológicas podridão apical e escaldadura em frutos de pimentão, submetido a diferentes tensões de água no solo $(-\mathrm{kPa})$ e doses de silício $\left(\mathrm{kg} \mathrm{ha}^{-1}\right)$ na forma de silicato de potássio via aplicação foliar (correlation coefficient between the incidence of physiological anomalies blossom-end rot and scald in sweet pepper fruits under different soil water tensions $(-\mathrm{kPa})$ and levels of silicon $\left(\mathrm{kg} \mathrm{ha}^{-1}\right)$ as potassium silicate application through leaves).Urutaí, IF Goiano, 2012.

\begin{tabular}{lcccc}
\hline Anomalia & $\begin{array}{c}\text { Número de } \\
\text { observações }\end{array}$ & Correlação & $\mathbf{Z}$ & $\mathbf{P}$ \\
\hline \multicolumn{5}{c}{ Tensão de água no solo } \\
\hline Podridão apical & 12 & $0,89^{*}$ & 2,9693 & 0,0015 \\
Escaldadura & 12 & 0,44 & 1,4846 & 0,0688 \\
\hline \multicolumn{5}{c}{ Doses de silício } \\
\hline Podridão apical & 12 & $-0,2324$ & $-0,7709$ & 0,2204 \\
Escaldadura & 12 & $-0,0726$ & $-0,2409$ & 0,4048 \\
\hline
\end{tabular}

$\mathrm{Z}=$ valor das médias da população; $\mathrm{P}=$ significância ao nível de $5 \%$ de probabilidade $(p<0,05) *$ valor significativo a $5 \%$ de probabilidade pela correlação de Spearman $[Z=$ value of population means; $\mathrm{P}=$ significance at $5 \%$ probability $(\mathrm{p}<0.05)$. ${ }^{*}$ significant value at $5 \%$ probability by Spearman correlation]. 


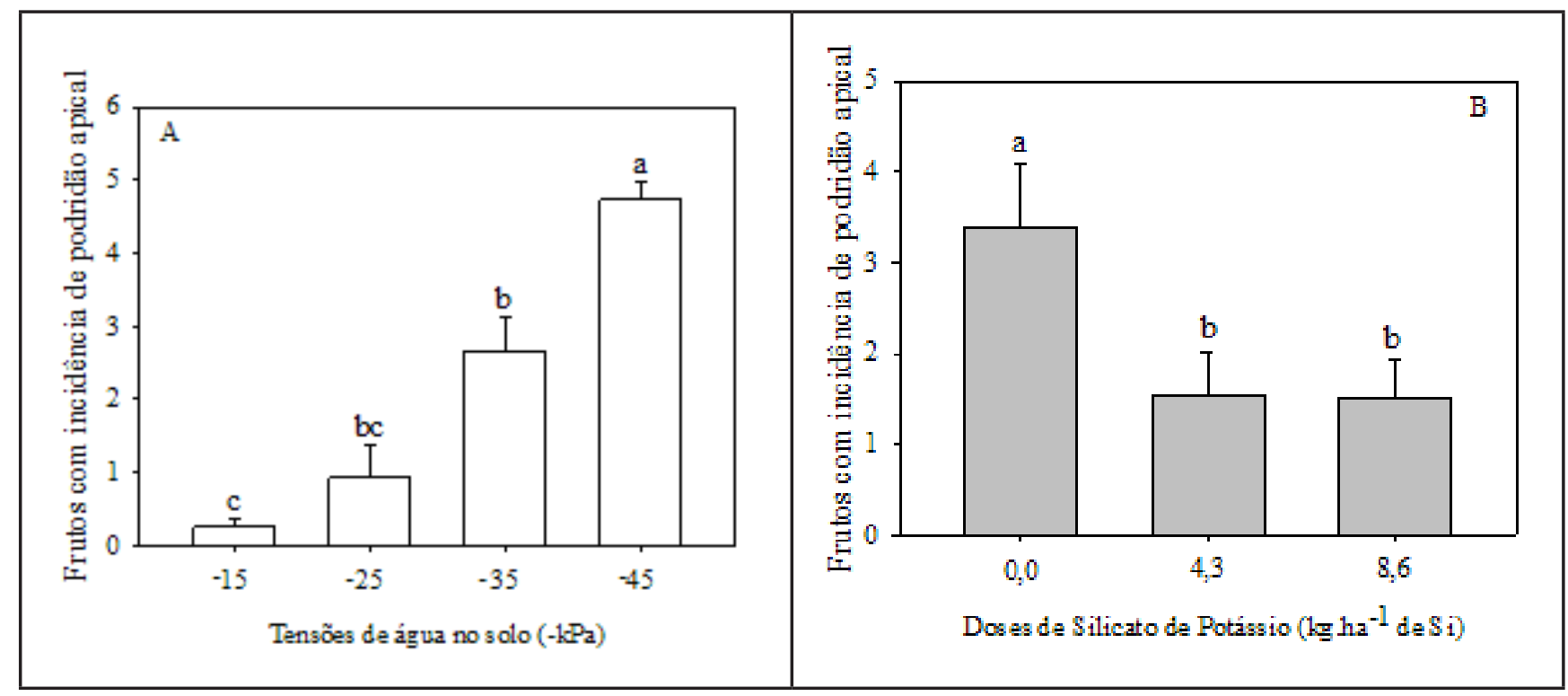

Figura 1. Número de frutos (média $\pm \mathrm{EP}$ ) de pimentão, com incidência de podridão apical colhidos in natura, em função do fator tensão de água no solo (-kPa) (A) e doses de silicio $\left(\mathrm{kg} \mathrm{ha}^{-1}\right)(\mathrm{B})$; médias seguidas pela mesma letra entre as barras, para ambas as figuras, não diferem entre sí pelo teste de Tukey ( $>0,05)$ [number of fruits (average \pm EP) of sweet pepper, with the incidence of blossom-end rot picked up in natura, due to the water tension in soil $(-\mathrm{kPa})(\mathrm{A})$ and levels of silicon $\left(\mathrm{kg} \mathrm{ha}^{-1}\right)(\mathrm{B})$; Average values followed by the same letter between the bars for both figures do not differ significantly from each other by Tukey test (p>0.05)]. Urutaí, IF Goiano, 2012.

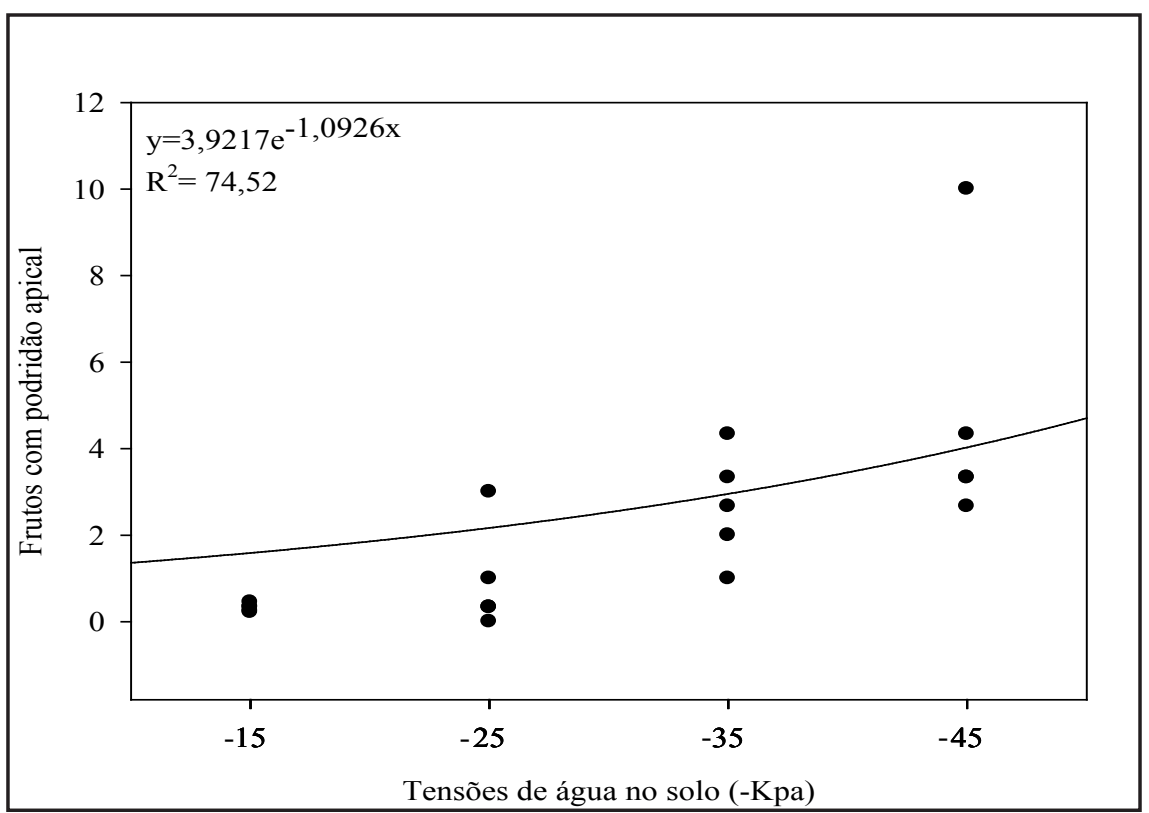

Figura 2. Correlação exponencial entre a incidência de podridão apical em frutos de pimentão, colhidos in natura, e tensões de água no solo (-kPa); *valores compreendidos pela média do número de frutos com podridão apical para cinco colheitas; valor significativo a $5 \%$ de probabilidade [logarithmic correlation between the incidence of blossom-end rot in sweet pepper fruits harvested in natura, and soil water tension $(-\mathrm{kPa})$; *values perceived by average of number of fruits with blossom-end rot for five harvest dates; significant value at 5\% probability). Urutaí, IF Goiano, 2012.

de silicato de potássio originaram frutos com maior incidência de podridão apical em número de frutos (Figuras $1 \mathrm{~A}$ e $\mathrm{B}$, respectivamente).

Apenas a correlação entre a tensão de água no solo e a incidência da podridão apical foi constatada como significativa e positiva no presente estudo (Tabela 1). Nesse caso, o número de frutos com sintoma de podridão apical tende a aumentar seguindo um modelo de regressão exponencial $(\mathrm{F}=40,96 ; \mathrm{p}=$ $0,0001)$ à medida que o valor da tensão de água no solo aumenta (Figura 2).

A incidência de podridão apical nos frutos de pimentão no presente trabalho, em virtude do aumento da tensão de água no solo, pode ser explicada pela deficiência hídrica durante a fase inicial de frutificação que restringe a translocação de cálcio nas plantas de pimentão (Filgueira, 2008; Souza et al., 2011). Sendo assim, as principais causas de desordem fisiológica em plantas que causa o aparecimento de podridão apical nos frutos são a baixa umidade no solo, maior intensidade de transpiração foliar e desequilíbrio nutricional, pois, influenciam a absorção, translocação e acúmulo de Ca nas plantas (Saure, 2001; Beninni et al., 2003; Terraza et al., 2008).

A adubação silicatada mostrou ter influência na redução do aparecimento da podridão apical indiferente do estado hídrico das plantas de pimentão. Essa redução pode ser entendida pelo fato do Si promover o uso mais eficiente da água (Carvalho et al., 2002) que demonstrou redução dos sintomas da podridão apical nos frutos de tomate utilizando adubação silicatada com aplicações via solo. Plantas de alface cultivadas em sistema 
hidropônico em solução com silício também apresentaram menores problemas com o surgimento de anomalias fisiológicas do tipo queima dos bordos, pois, o Si aumenta a rigidez das células das plantas e regula a transpiração (Luz et al., 2006). A aplicação foliar de Si, apesar de ser considera menos eficiente que aplicação via solo, e na solução hidropônica, também comprovam suas vantagens em algumas culturas.

As plantas de pimentão não sofreram perdas significativas da cobertura foliar decorrente do estado hídrico deficitário imposto no presente trabalho; esse motivo pode explicar a não significância do surgimento da anomalia escaldadura, pois segundo Filgueira (2008), a queda das folhas e a exposição direta dos frutos à luz solar é o principal causador da escaldadura em frutos de pimentão.

\section{AGRADECIMENTOS}

Aos colaboradores, Gaspar Henrique Korndörfer, Fabrício de Ávila Rodrigues e Instituto Federal Goiano, câmpus Urutaí.

\section{REFERÊNCIAS}

BENINNI ERY; TAKAHASHI HW; NEVES CSVJ. 2003. Manejo de cálcio em alface de cultivo hidropônico. Horticultura Brasileira 21: 605-610.

BUCK GB; KORNDÖFER GH; NOLLA A; COELHO L. 2008. Potassium silicate as foliar spray and rice blast control, Journal of Plant Nutrition 31: 231-237.

CARVALHO JG; MACHADO AQ; NASCIMENTO IR; BOAS RCV. 2002. Desempenho da cultura do tomate adubado com silifértil. Horticultura Brasileira 20: 2, Suplemento 2.
CRUSCIOL CAC; PULZ AL; LEMOS LB; SORATTO RP; LIMA GPP. 2009. Effects of silicon and drought stress on tuber yield and leaf biochemical characteristics in potato. Crop Science 49:949-954.

FERREIRA PV. 2000. Estatística experimental aplicada à agronomia. Maceió. EDUFAL. 419p.

FILGUEIRA FAR. 2008. Novo manual de olericultura: agrotecnologia moderna na produção e comercialização de hortaliças. 3 ed. Viçosa. UFV. 421p.

GONÇALVES VG. 2009. Arquitetura de planta, teores de clorofila e produtividade de batata, cv. Atlantic, sob doses de silicato de potássio via foliar. Uberlândia: UFU. 51p (Dissertação mestrado).

GONG H; ZHU X; CHEN K; WANG S; ZHANG C. 2005. Silicon alleviates oxidative damage of wheat plants in pots under drought. Plant Science 169: 313-321.

GUNES A; INAL A; BAGCI EG; COBAN S. 2007. Influence of silicon on antioxidant mechanisms and lilip peroxidation in chickpea (Cicer arietinum L.) cultivars under drought stress. Journal of Plant Interactions 2: 105113.

HARTZ TK; CANTWELL M; LESTRANGE M; SMITH RF; AGUIAR J; DAUGOVISH O. 2008. Bell peper production in California. Oakland: University of California. 4p (Vegetable Production Series. Publication, 7217).

KORNDÖRFER GH; PEREIRAHS; CAMARGO MS. 2002. Silicato de cálcio e magnésio na agricultura. Uberlândia: UFU/ICIAG, $3 p$ (GPSi-ICIG-UFU. Boletim Técnico, 01).

LIANG YC; SUN WC; SI J. 2005. Effects of foliar- and root-applied silicon on the enhancement of induced resistance to powdery mildew in Cucumis sativus. Plant Pathology 54: 678-685.

LUZ JMQ; GUIMARÃES STMR; KORNDÖRFER GH. 2006. Produção hidropônica de alface em solução nutritiva com e sem silício. Horticultura Brasileira 24: 295-300.

MA JF; YAMAJI N. 2006. Silicon uptake and accumulation in higher plants. Trends in Plant Science 11: 392-397.

MENZIES JG; EHRET DL; GLASS ADM; HELMER T; KOCH C; SEYWERD F. 1991. The influence of silicon on cytological interactions between Sphaerotheca fuliginea and Cucumis sativus. Physiology Molecular Plant Pathology 39: 403-414.

NOLLA A; FARIA RJ; KORNDÖRFER GH; SILVA TRB. 2012. Effect of silicon on drought tolerance of unpland rice. Journal of Food, Agriculture \& Environment 10: 269-272.

POZZA AAA; ALVES E; POZZA EA; CARVALHO JG; MONTANARI M; GUIMARÃES PTG; SANTOS DM. 2004. Efeito do silício no controle da cercosporiose em três variedades de cafeeiro. Fitopatologia Brasileira 29: 185-188.

RIBEIRO JUNIOR JI; MELO ALP. 2009. Guia prático para utilização do SAEG. Viçosa. UFV. 288p.

RODRIGUES CR; RODRIGUES TM; FIGUEIREDO FC. 2007. Aplicação de silício líquido solúvel via foliar em batata: Aumento de 5 toneladas/ha de batata extra. Revista Campo e Negócios HF: 10: 66-70.

RODRIGUES FA; DUARTE HSS; REZENDE DC; WORDELL FILHO JA; KORNDÖRFER GH; ZAMBOLIM L. 2010. Foliar spray of potassium silicate on the control of angular leaf spot on beans. Journal of Plant Nutrition 33: 2082-2093.

SAURE MC. 2001. Review. Blossom-end rot of tomato (Lycopersicon esculentum) a calcium- or a stress-related disorder? Scientia Horticulture 90: 193-208.

SOUZA VF; MAROUELLI WA; COELHO EF; PINTO JM; COELHO FILHO MA. 2011. Irrigação e fertirrigação em fruteira e hortaliças. Brasília: Embrapa 721-736p. (Informação Tecnológica).

TERRAZA SP; ROMERO MV; PEÑA PS; MADRID JLC; VERDUGO SH. 2008. Efecto del calcio y potencial osmótico de la solución nutritiva em la pudrición apical, composición mineral y rendimento de tomate. Interciencia 33: 449-456.

TRANI PE. 2007. Calagem e adubação para hortaliças sob cultivo protegido. Artigo em Hypertexto. Disponível em: <http://www. infobibos.com/Artigos/2007_1/cp/index. $\mathrm{htm}>$. Acesso em: 11/6/2011.

TRANI PE; CARRIJO OA. 2004. Fertirrigação em hortaliças. Campinas: Instituto Agronômico. 58 p.

VIDHYASEKARAN P. 1997. Fungal pathogenesis in plants and crops molecular biology and host defense mechanisms. New York: Marcel Dekker. 